Provided for non-commercial research and education use. Not for reproduction, distribution or commercial use.

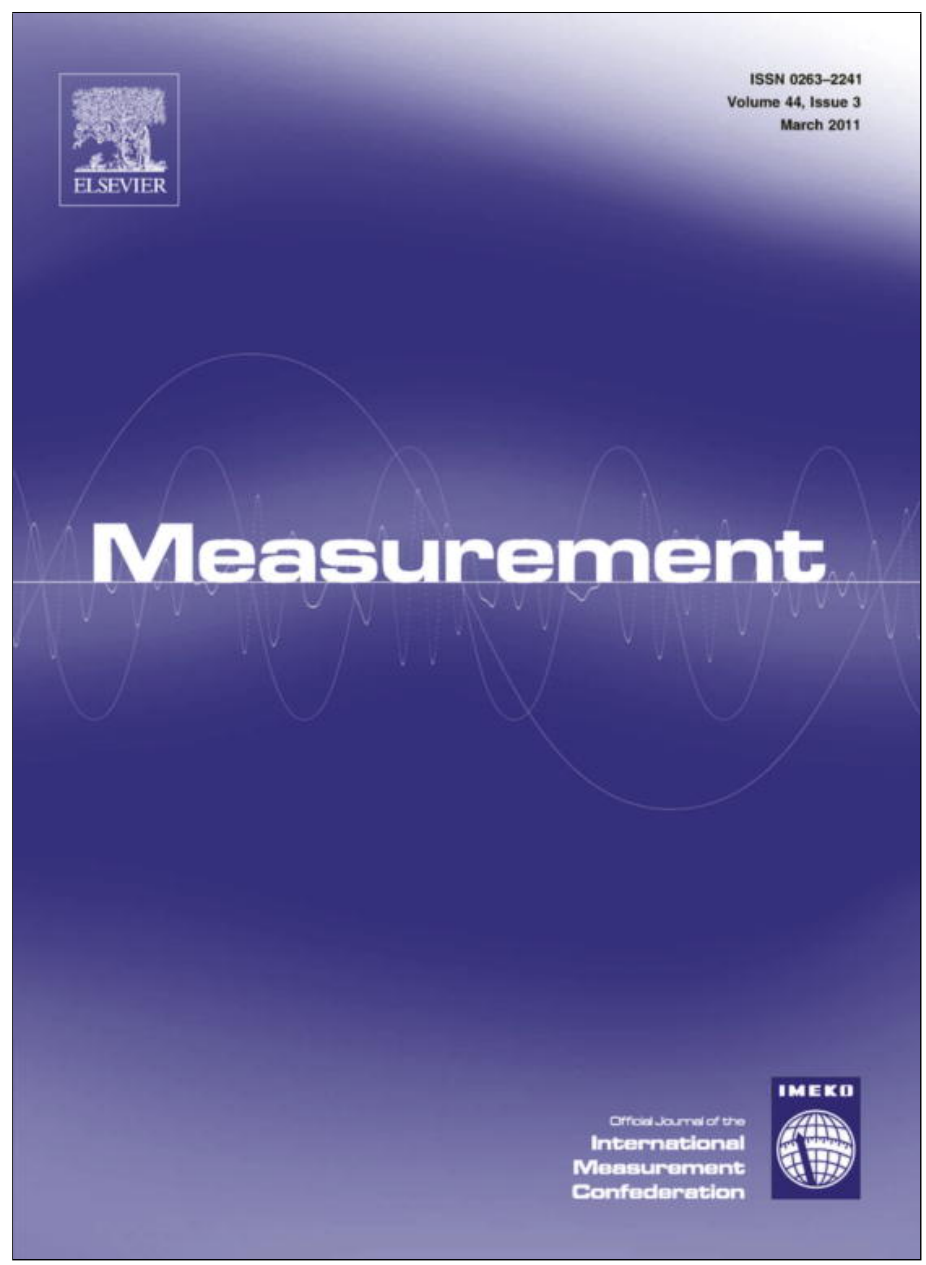

This article appeared in a journal published by Elsevier. The attached copy is furnished to the author for internal non-commercial research and education use, including for instruction at the authors institution and sharing with colleagues.

Other uses, including reproduction and distribution, or selling or licensing copies, or posting to personal, institutional or third party websites are prohibited.

In most cases authors are permitted to post their version of the article (e.g. in Word or Tex form) to their personal website or institutional repository. Authors requiring further information regarding Elsevier's archiving and manuscript policies are encouraged to visit:

http://www.elsevier.com/copyright 


\title{
Residual stress measurement in PVD optical coatings by microtopography
}

\author{
Manuel F.M. Costa*, V. Teixeira \\ Universidade do Minho, Departamento de Física, Campus de Gualtar, 4710 - 057 Braga, Portugal
}

\section{A R T I C L E I N F O}

\section{Article history:}

Received 1 July 2010

Received in revised form 11 November 2010

Accepted 17 November 2010

Available online 25 November 2010

\section{Keywords:}

Residual stress

Microtopography

Thin films

PVD

$\mathrm{WO}_{3}$

Triangulation

\begin{abstract}
A B S T R A C T
Residual stress in optical plasma vapor deposited coatings must be carefully measured. The topographic inspection of the coatings' surface at microlevel allows the assessment of its residual stress. In the present work we will report on the optical non-destructive and non-invasive microtopographic inspection of $\mathrm{WO}_{3} \mathrm{PVD}$ thin films for residual stress evaluation. The MICROTOP.06.MFC system, an active optical triangulation sensor developed at the Universidade do Minho, was employed. It allows depth resolutions down to $2 \mathrm{~nm}$ and lateral resolutions down to $1 \mu \mathrm{m}$. The three dimensional coordinate set obtained on the inspection allow the calculation of the stress distribution over the film.
\end{abstract}

(c) 2010 Elsevier Ltd. All rights reserved.

\section{Residual stress measurement by microdisplacement techniques}

Residual stress of as-deposited PVD coatings may cause bending of the coating/substrate system. If residual stresses are present and the overall deflection is small compared with the substrate thickness, then by symmetry the coated substrate will take up a spherical curvature in the region away from the edges [1]. Near the edges a complex stress state will be present. However away from the edges this reverts to a simple stress state where stresses normal to the substrate and shear stresses are zero [2-4]. Therefore we will measure the residual stress of the coatings from the radius of curvature of the coating/substrate system measured away from the edges.

In Fig. 1 we illustrate the typical measurement of the radius of curvature of the PVD coating/substrate system. Since the deflections (a few microns) is several orders of magnitude smaller than the sample length (up to a few centimeters), its circular profile approximates to a parabola (Fig. 2). Therefore, the height values were fitted to a

\footnotetext{
* Corresponding author. Fax: +351253604061.

E-mail address: mfcosta@fisica.uminho.pt (M.F.M. Costa).
}

general parabolic equation by a least-squares procedure and thus the radius of curvature in the middle of the coating can be calculated from the fitted parameters. This method is much less prone to inaccuracies than simply measuring the height difference between the middle and ends of the sample. Slight discrepancies of data point compared with the fitting parabolic curve may be caused by positioning errors of the coated substrate under the laser beam but this is a minor random error which is likely to average out along the sample, giving the same fitting parameters.

Once the radius of curvature is determined, and since the PVD coating is thin compared with the substrate thickness, the in-plane residual stress in the coating, $\sigma_{\mathrm{c}}$, can be determined as per the equation [1-6]:

$\sigma_{\mathrm{c}}=\frac{E_{\mathrm{s}}}{6\left(1-v_{\mathrm{s}}\right)} \frac{t_{\mathrm{s}}^{2}}{t_{\mathrm{c}}}\left(\frac{1}{R_{\mathrm{c}}}-\frac{1}{R_{\mathrm{uc}}}\right)$

where $E_{\mathrm{s}}$ is the Young's modulus of substrate, $v_{\mathrm{s}}$ is the Poisson ratio of substrate, $R_{\mathrm{uc}}$ is the radius of the curvature of the uncoated substrate, $R_{\mathrm{c}}$ the radius of curvature of the coated substrate, and $t_{\mathrm{s}}$ and $t_{\mathrm{c}}$ are the substrate and coating thickness, respectively. The first term in Eq. (1) is used to correct for non-flat substrates before the deposition of 


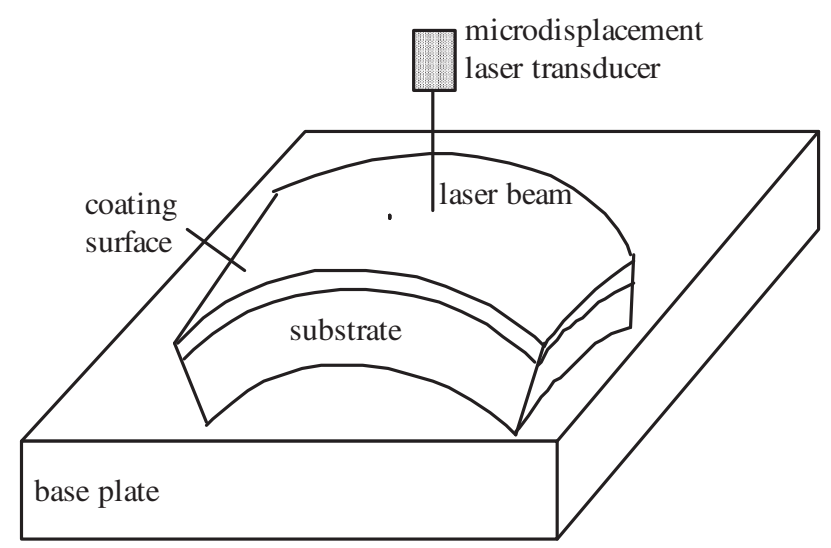

Fig. 1. Schematic representation of the curvature measurements in the as-deposited PVD coatings. The laser transducer is fixed and the coating/ substrate system is moved with the coating surface facing up, as shown. The base plate is then traversed under the laser beam and surface height measurements are made at regular intervals along the center-line of the sample [2].

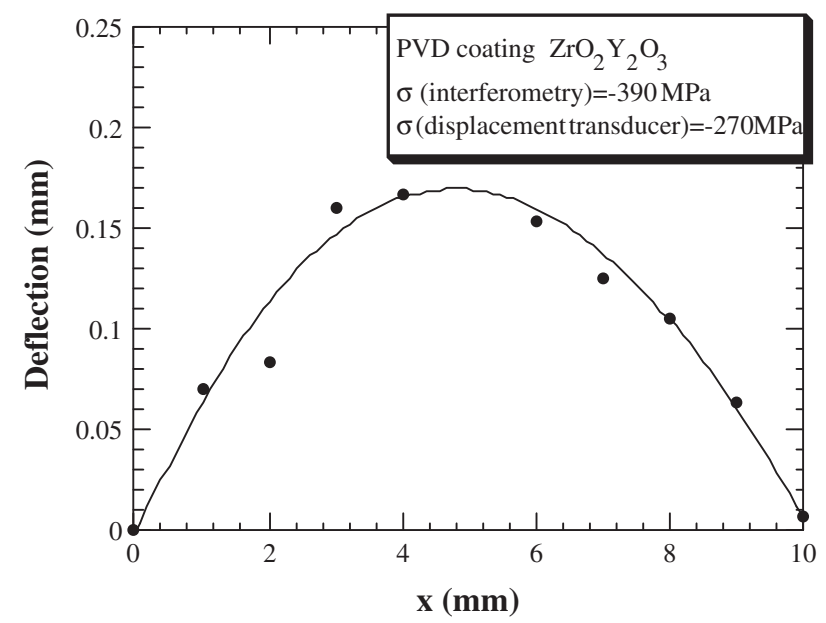

Fig. 2. Typical parabolic profile for a PVD coating (yttria stabilized zirconia), measured by the microdisplacement laser transducer ( $x$ is the position along the center-line of the coating-substrate as shown in Fig. 1) [3].

the coating. The radius of curvature can also be determined by other methods such as interferometry [7], where the interference fringes in a finished optical surface (such as a glass substrate) are used to obtain the curvature in the bent sample. In the present work we used a laser triangulation based microtopographer developed at the Microtopography Laboratory of the Physics Department of the University of Minho [7,8]. Being a 3D inspection system we are able to measure curvatures not only from profiles but yes tridimensionally from the relief maps obtained. The method and system is described and a few results of residual stress measures on $\mathrm{WO}_{3}$ thin films on glass substrates is presented below.

\section{The microtopographic inspection system}

Optical triangulation [9], as a non-contact non-destructive method, extensively proved its usefulness on the topo- graphic and dimensional inspection of objects and surfaces of use in the industrial world. Not only rough surfaces [10] can be microtopographically inspected [7,8]. Also the inspection of smooth mirror like surfaces is possible with resolutions in the nanometer range $[11,12]$.

The method over which we based our work is optical active triangulation with oblique incidence and normal (and or specular) observation, and automated mechanical sample's scanning [8]. The surface to be inspected is scanned by an oblique laser beam. The incident light is collimated and focused. A small, diffraction limited, bright spot is thus projected onto the sample. The bright spot is imaged perpendicularly and specularly onto electronic photosensitive detection systems in order to assess its lateral position. The area of the surface to be inspected is scanned point-by-point by the "sensor's tip" (the light beam focused onto the surface). The highest system's robustness was sought. Also a high lateral positioning resolution and accuracy is achieved. In order to perform the sample's scanning it will be moved by means of a precision $\mathrm{XY}$ displacement table driven by precision step motors. At each scanning point the lateral spot's position is obtained, using both, or just one of the detection systems of the normal and specular reflection arms of the sensor. For mirror like types of surfaces the more reliable data is obtained with the specular data while for very rough surfaces the specular arm does not give reliable enough depth measures. The horizontal spot's shift between scan positions is directly related to the height differences between those surface's inspected points and thus the topographic map is obtained.

The depth resolution depends of the system used and on its particular configuration. The MICROTOP.03.MFC $[7,8]$ is a robust and versatile system specially designed to accurately perform the microtopographic inspection of the rough surface of small samples. It allows the inspection of a large variety of surfaces with resolutions that can be driven down to the submicron level with dynamic ranges up to $1: 5000$ (or $1: 25,000$ with vertical scanning).

In order to be able to inspect smaller features in smoother surfaces a different version of this microtopographer was settled. The MICROTOP.06.MFC [12,13] maintain the main characteristic of its predecessor gaining an increased versatility with the incorporation of a second specular reflection arm. Now, height measures on mirror like surfaces or optically non-rough surfaces can be performed with accuracy in the nanometer range and resolution in the sub-nanometer range with dynamic ranges up to $1: 20,000$ with proper vibration isolation. The inspection of a large variety of thin films and coatings is then possible with good resolution and reliability.

The MICROTOP.06.MFC microtopographer is based, as said above, on active optical triangulation and angle resolved scattering. Referring to Fig. 3, a brief description is given below. The surface to be inspected is scanned by an oblique light beam. Different light sources both coherent and incoherent are used according to the type of sample to be inspected: two HeNe lasers at 632.8 and $534 \mathrm{~nm}$, and, one Xe white light sources are available and can be easily interchanged. The incident light is collimated and focused. A small, diffraction limited, bright spot is thus 


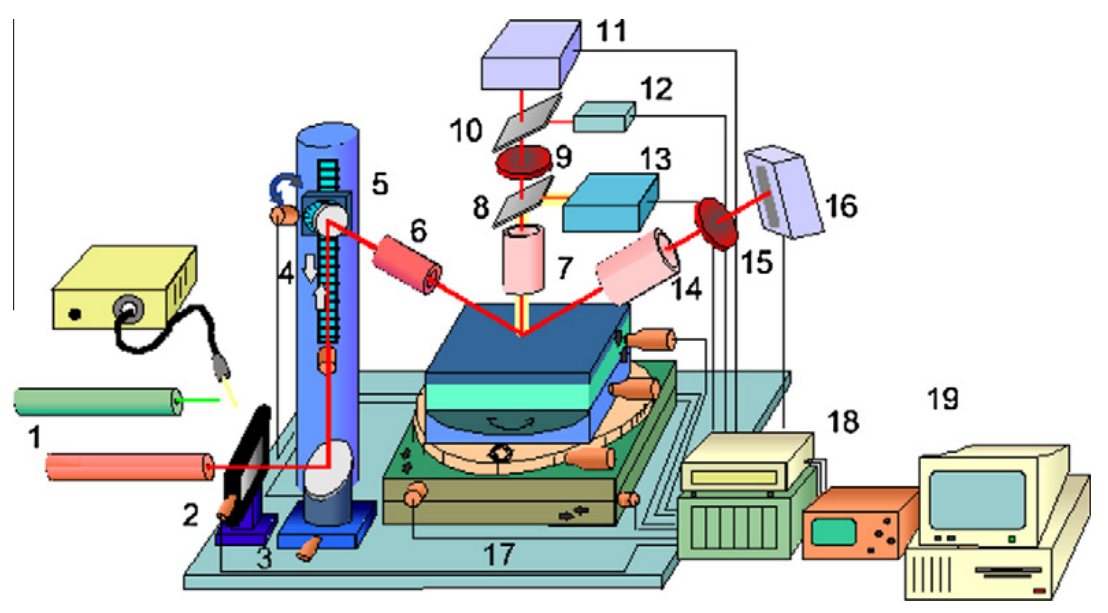

Fig. 3. The MICROTOP.06.MFC: 1. Interchangeable light sources; 2 . Vibration isolation stand; 3 . Neutral density filter; 4 . Beam steering system; 5. Incidence angle control motorised system; 6 . Incidence optics; 7 . Normal observation optics; 8 and 10 . Beam splitters; 9 . Interference filter; 11 . Normal photosensitive detection system; 12. Photodetector; 13 . Video camera and illuminator; 14 . Specular observation optics; 15 . Interference filter; 16 . Specular photosensitive detection system; 17. Sample support and motorised positioning system; 18. Data acquisition and control system; 19. Microcomputer.

projected onto the sample. The bright spot is imaged both perpendicularly and specularly onto electronic photosensitive detection systems in order to assess its lateral position. The photosensors are one 2048 pixels Fairchild CCD linear array on the specular arm and a Reticon line scan camera. However, one PSD, position sensitive detector (from Sitek) and a differential detector (from Hammamatsu) are available and can easily replace the linear arrays. The area of the surface to be inspected is scanned point-by-point by the "sensor's tip" (the light beam focused onto the surface). The highest system's robustness was sought. Also a high lateral positioning resolution and accuracy should be achieved. Thus both the incidence arm and observation arms of the sensor are kept fixed. In order to perform the sample's scanning it will be moved by means of a precision $\mathrm{XY}$ displacement table driven by precision step motors (General Electric). At each scanning point, on a rectangular array separated by distances down to $1.25 \mu \mathrm{m}$, the lateral spot's position in both sensors is obtained and registered. The spot's shift on both detectors' planes, between consecutive scan positions is directly related with the height differences between those surface' inspected points. When necessary in the "specular" arm of the system the detector can be positioned (just by introducing an adapter) tilted relative to the observation optics in order to increase the depth range of the sensor (Schleimpflug' condition) [7]. Employing the linear arrays both arms are on a confocal arrangement allowing the best resolution [7]. The incidence set-up comprises apart from the light source a neutral density variable filter, a stabilized beam steering system, a spatial filter and focusing optics. The change on the incidence angle is made synchronized with the change of the observation angle on the specular arm. A vertical movement precision stage endowed of computer controlled motion provided by a reliable accurate DC encoder with high positioning repeatability and resolution is used refocusing of the observation optical system but especially for calibration of both arms of the sensor. In order to resolve shaded areas and mutual reflections, a high precision rotational stage is used allowing easy change to opposite light incidence. Often the faces of the surface to be analyzed are not parallel or simply the surface to be inspected does not lie horizontally. In order to maintain the best height resolution a tilt table was incorporated to the samples' positioning system. Furthermore it may allow the inspection of 3D objects or surfaces with pronounced holes of it, for instance.

The observation optical systems are formed by microscope objectives chosen according to the characteristics of the surface's relief. In both sensor' arms the objectives can be independently focused. They will be used to image the light spot onto the opto-electronic photosensitive detection systems. Both the "normal" and the "specular" sensors' arms are attached to a XYZ precision displacement table for finer adjustments. A 2D CCD camera was attached to the system allowing the capture of bidimensional color images of the scanned area for matching and improved visualization aid. Projection of the actual 2D image onto the $3 \mathrm{D}$ map is being studied at the moment. In order to cope with different requirements different photosensitive systems are available and all are interchangeable (see above). A personal microcomputer acquires the data and takes control of the whole inspection process and result's presentation. At the end of the inspection process we may have just one but typically will have two sets of data one for each sensor's arm. Data processing is independently performed and two sets of parameters and functions are obtained by triangulation and scattering analysis. The correlation of the sets of data is investigated. Comparison and matching is performed in order to obtain the best set of reliable and accurate data. Depth resolutions down to $2 \mathrm{~nm}$ and lateral resolutions down to $1 \mu \mathrm{m}$ with dynamic ranges of $1: 25,000$ and measurement speeds of 2000 points per second are achieved.

\section{Results}

From the coatings surfaces' relief maps obtained with our laser microtopographer we are able to calculate the 
coatings' residual stress in that particular coating/substrate system.
In Fig. 4 we present the results of the stress evaluation of a $123 \mathrm{~nm}$ thick $\beta$ tungsten thin film PVD deposited, on a

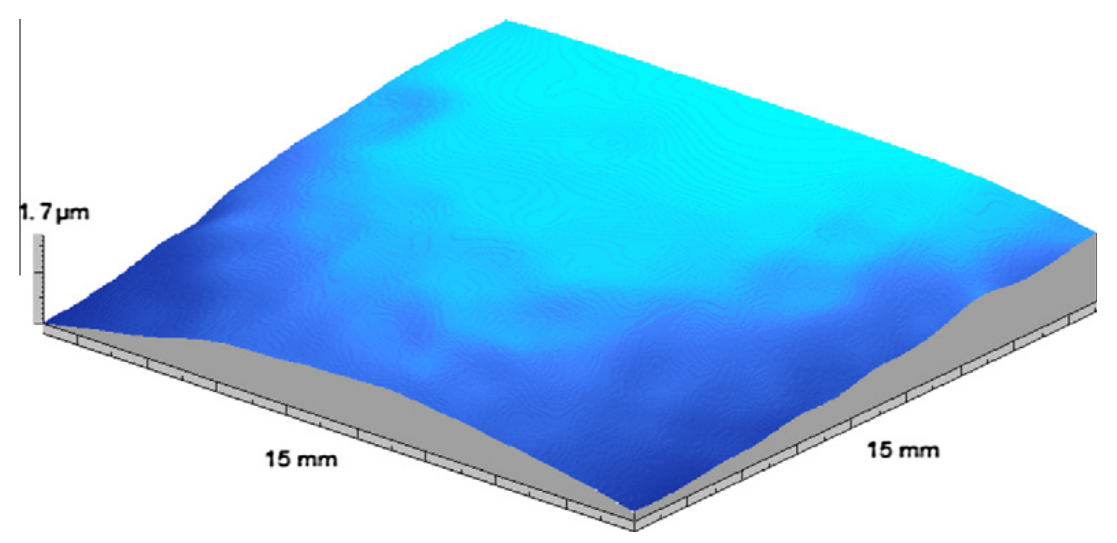

Fig. 4. Relief map of a $\mathrm{WO}_{3}$ film used in electrochromic applications [10].

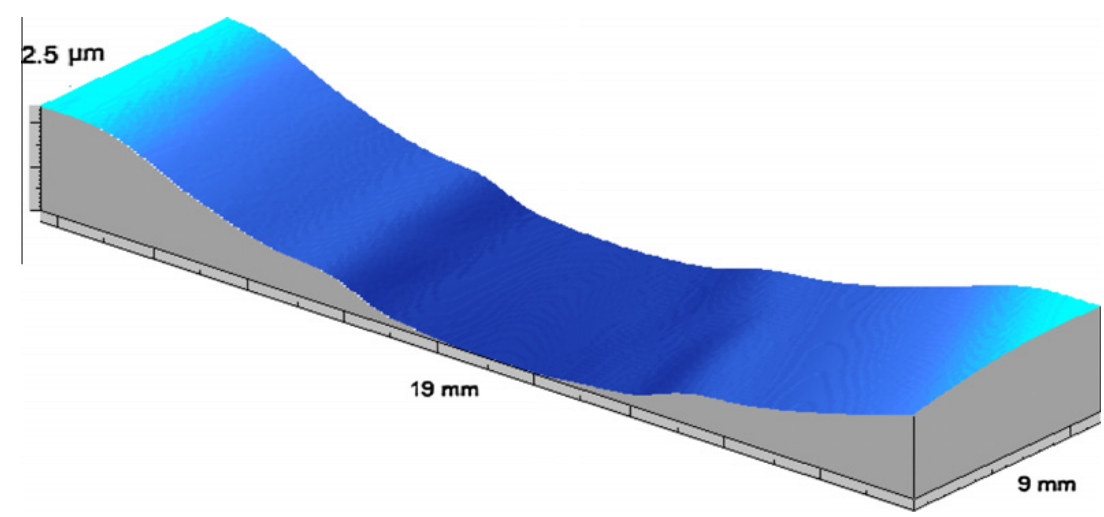

Fig. 5. Microtopographic map of a tungsten film. Different curvatures in central and outer regions of the film are noticeable possibly due to partial nonadherence of the film to the substrate.
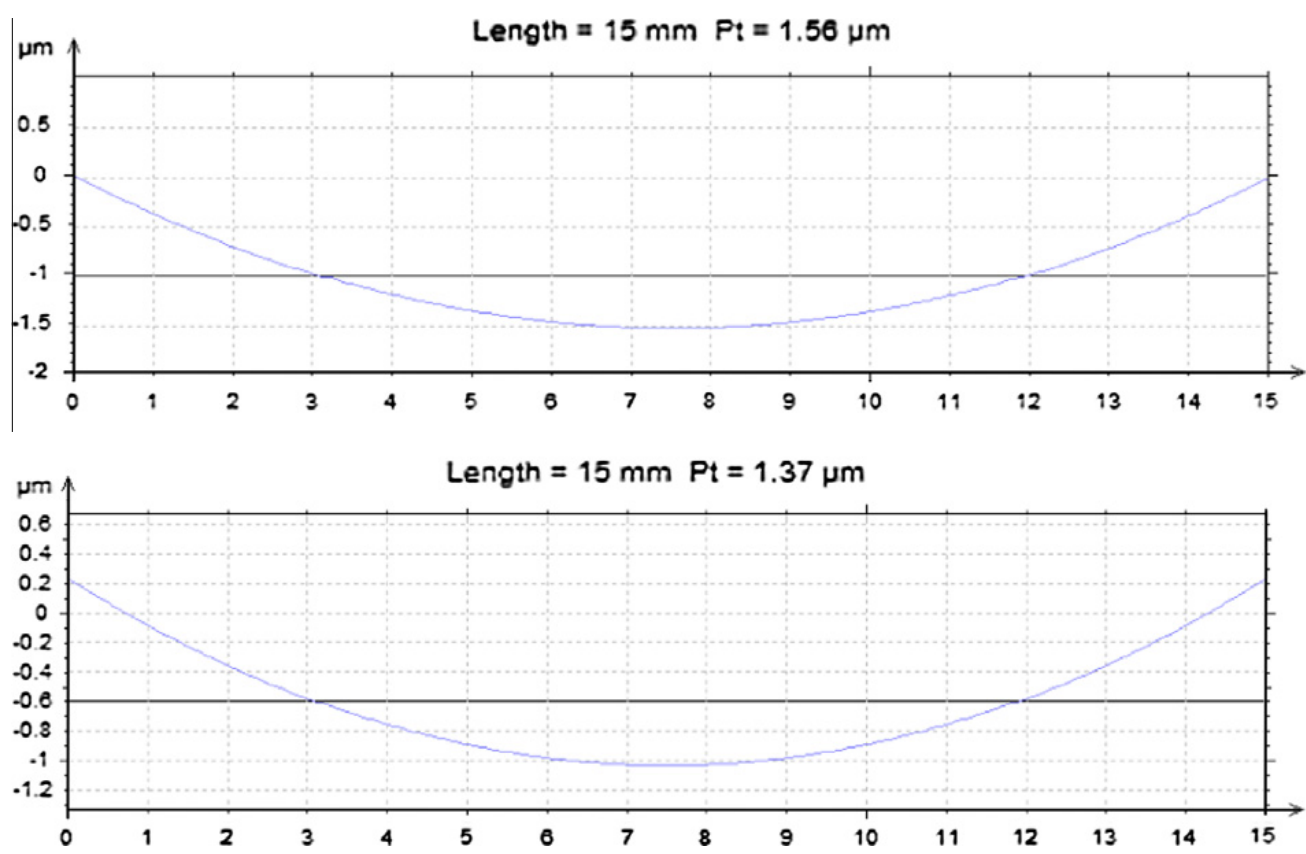

Fig. 6. Principle parabolic profiles of the tungsten film whose 3D relief map is presented in Fig. 5 fitted on the central (above) and outer regions of the film (below). 
$15 \mathrm{~mm}$ per $15 \mathrm{~mm}$ square glass blade $0.15 \mathrm{~mm}$ thick, at a $4 \times 10^{-2}$ mbar pressure during 6 min in a sputtering chamber. The substrate-target distance was set to $60 \mathrm{~mm}$ and the sputtering current of $0.23 \mathrm{~A}$. The thin film (in fact the film/substrate system) presents a slight curvature and a surface with an average roughness (Ra) [7] of $0.34 \mu \mathrm{m}$. The calculated transversal compressive stress ranges between 461 and $447 \mathrm{MPa}(454.38 \pm 6.84 \mathrm{MPa})$ across the central region of the film. This roughly $1.5 \%$ variation is not significant and the stress distribution essentially isotropic. The three-dimensional topographic inspection of the sample allows a easier detection of any anisotropy in the residual stress distribution. Furthermore we can also distinguish zones in the coating that may eventually present different residual stress for instance off the central region of the film. In Fig. 5 is shown a thin film that produced a major curvature in the glass substrate. High stress caused a partial non-adherence of the film to the substrate. Nevertheless being evident the detaching the residual stress calculated in the central zone (still adhered to the substrate) is only 9\% higher than at outer regions (510.7 and 459.8 MPa: Fig. 6 above and below respectively).

\section{Conclusions}

Residual stress is a major parameter in the characterization of thin films. Different methods exists and are successfully used in residual stress measurement in thin films and coatings of different types [1,4,14-16].

Contactless methods allowing non-invasive stress measures are advisable in many instances. Optical distance sensors and profilometers may be used to measure curvatures of thin films bended due to stress allowing a non-destructive non-contact evaluation. Integral microtopographic inspection of the surface of PVD coatings and thin films in different substrates allow a better assessment of its residual stress.

The performance of our non-invasive optical microtopographer allows a reliable and efficient inspection of the PVD optical coatings in order to detect flaws and defects, to evaluate the films roughness but also to determine stress distribution on the produced films in a non-invasive fast, accurate and reliable way. Due to the robustness of the optical triangulation procedure used, a large range of thin film' surfaces from polished mirror like to rough ones can be measured. The measurement process does not allow in situ evaluation of the samples. Furthermore the inspection process being point-by-point is inherently relatively slow (up to 2000 inspected points per second) yet with high lateral and height resolution and repeatability. The employed system' configuration can me adjusted according to the particular sample characteristics allowing the measure of samples of different sizes and grades of stress induced deformations The 3D representation of the films' surface allows the identification of stress zones in nonhomogeneous films.

\section{References}

[1] P.A. Flinn, D.S. Gardner, W.D. Nix, Measurement and interpretation of stress in aluminum-based metallization as a function of thermal history, IEEE Trans. Electron. Dev. ED-34 (13) (1987) 689.

[2] V. Teixeira, Mechanical integrity in PVD Coatings due to the presence of residual stresses, Thin Solid Films 392 (2001) 276-281.

[3] P. Gao, L.J. Meng, M.P. dos Santos, V. Teixeira, M. Andritschky, Influence of sputtering power and the substrate-target distance on the properties of $\mathrm{ZrO}_{2}$ films prepared by $\mathrm{RF}$ reactive sputtering, Thin Solid Films 377 (2000) 557.

[4] K.-S. Chen, K.-S. Ou, Modification of curvature-based thin film residual stress measurement for MEMS applications, J. Micromech. Microeng. 12 (2002) 917

[5] V. Teixeira, M. Andritschky, Residual stress in coatings produced by magnetron. Sputtering, High Temp. High Press. 25 (1993) 213.

[6] E. Fortunato, D. Brida, L. Pereira, H. Águas, V. Silva, I. Ferreira, M.F.M. Costa, V. Teixeira, R. Martins, Dependence of the strains and residual mechanical stresses on the performances presented by a-Si:H thin film position sensors, Adv. Eng. Mater. 4 (8) (2002) 612-616.

[7] Manuel F.M. Costa, J.B. Almeida, System of optical non-contact microtopography, Appl. Opt. 32 (1993) 4860-4863.

[8] Manuel F.M. Costa, Surface inspection by an optical triangulation method, Opt. Eng. 35 (9) (1996) 2743-2747.

[9] G. Seitz, H. Tiziani, R. Litschel, 3D coordinates measurement by optical triangulation, F M-Feinwerktech. Mes. 94 (7) (1986) 423425.

[10] Z. Ji, M.C. Leu, Design of optical triangulation devices, Opt. Laser Technol. 21 (5) (1989) 335-338.

[11] A. Monteiro, M.F. Costa, B. Almeida, V. Teixeira, J. Gago, E. Roman, Structural and optical characterization of $\mathrm{WO}_{3}$ deposited on glass and ITO, Vacuum 64 (2002) 287.

[12] C. Batista, A. Portinha, R.M. Ribeiro, V. Teixeira, M.F. Costa, C.R. Oliveira, Morphological and microstructural characterization of laser-glazed plasma-sprayed thermal barrier coatings, Surf. Coat. Technol. 200 (90) (2006) 2929-2937.

[13] H.N. Cui, M.F. Costa, V. Teixeira, J. Zhang, Solution-grown method of CdS film by ultrasonical colloid chemistry deposition technique. Int. J. Photoenergy, 2006, (article ID 24916), (2006) 4.

[14] Xin Zhang, Tong-Yi Zhang, Yitshak Zohar, Measurement of residual stresses in thin films using micro-rotating-structures, Thin Solid Films 335 (1998) 97-105.

[15] G. Sarau, M. Becker, G. Andrä, S. Christiansen, Residual stress measurements in multicrystalline silicon bulk and thin film solar cells using micro-Raman spectroscopy, in: 23rd Eur. Photovoltaic Solar Energy Conf., 1-5 September 2008, Valencia, Spain, 2008, pp 2265-2270.

[16] Q. He, Z.X. Luo, X.Y. Chena, Comparison of residual stress measurement in thin films using surface micromachining method, Thin Solid Films 516 (16) (2008) 5318-5532. 